\title{
Variation in Bordetella bronchiseptica flaA does not correlate with typing by macro-restriction analysis by pulsed-field gel electrophoresis
}

\author{
C. WINSTANLEY, A. SHINA, S. DAWSON*广, R.M. GASKELL* and C. A. HART. \\ Departments of Medical Microbiology and Genito-Urinary Medicine, *Veterinary Pathology and $\uparrow$ Veterinary \\ Clinical Science and Animal Husbandry, University of Liverpool, PO Box 147, Liverpool L69 $3 G A$
}

\begin{abstract}
A genotyping method based on PCR-RFLP analysis of the flagellin gene ( $f$ a $A$ ) was applied to 30 mainly feline isolates of Bordetella bronchiseptica. These isolates were separated into three PCR-RFLP groups with the restriction endonucleases Hae III, Msp I, MboI and RsaI. $\boldsymbol{f l a A}$ nucleotide sequences representing each of the three groups differed from each other by 11-13\%. One of the groups exhibited far greater fla $A$ sequence identity with the cryptic flagellin gene sequence of $B$. pertussis $(>97 \%)$ than with $f l a A$ sequences from representatives of the other $B$. bronchiseptica PCR-RFLP groups. Amongst the 30 isolates were at least 10 representing each of the two major genotypes ( $A$ and $B$ ) identified in a previous study by macro-restriction analysis and pulsed-field gel electrophoresis (PFGE), as well as representatives of other less common genotypes. Each of the major PFGE genotypes contained strains representing more than one flagellin genotype. Indeed, there was no correlation between the two molecular typing methods. PFGE analysis may identify differences due to genomic re-arrangements rather than genuine variations in gene content. If so, relationships inferred on the basis of PFGE or other molecular methods for whole genome comparison should be treated with caution.
\end{abstract}

\section{Introduction}

Bordetella bronchiseptica is a respiratory pathogen of a wide range of domestic and other animals [1] and has also been associated with human infections [2]. It has been recognised as a primary pathogen in canine kennel cough [3] and respiratory disease in cats [4-6]. A number of molecular methods for strain discrimination has been developed to study the epidemiology of B. bronchiseptica. These include randomly amplified polymorphic DNA (RAPD) fingerprinting [7], ribotyping [8] and macro-restriction analysis by pulsed-field gel electrophoresis (PFGE) [9, 10]. A study of a collection of 164 (predominantly feline) isolates of $B$. bronchiseptica by PFGE following macro-restriction digestion with the endonuclease $X b a$ I concluded that although the isolates fell into 17 different strains, 126 of these isolates were assigned to one of two strains (named A and B) [10]. Isolates were classified as identical if they showed no band differences, as subtypes of the same strain if they showed one-to-

Received 7 June 2000; accepted 2 Aug. 2000.

Corresponding author: Dr C. Winstanley

(e-mail: C.Winstanley@liverpool.ac.uk). three band differences, and as different strains if they showed four or more band differences (corresponding to $\leqslant 83 \%$ similarity).

Flagella, consisting of basal body, rod and filament, are the organelles of motility in many bacterial pathogens, including B. bronchiseptica. It has been suggested that flagella may also contribute to the adherence of $B$. bronchiseptica to eukaryotic cells [11]. The genus Bordetella contains both motile (B. bronchiseptica and $B$. avium) and non-motile (B. pertussis and $B$. parapertussis) species. However, all four of these species contain DNA sequences homologous to genes encoding the major filament protein, flagellin [12]. The presence of a non-expressed flagellin gene in the $B$. pertussis strain Tomaha I has been confirmed by genome sequencing (data can be obtained from ftp:// ftp.sanger.ac.uk/pub). The BvgAS signal transduction system of $B$. bronchiseptica modulates motility as well as a number of well characterised virulence factors, such as filamentous haemagglutinin, pili and adenylate cyclase toxin $[13,14]$. The flagellin gene $(f a A)$ is known to be negatively regulated by the Bvg regulon such that when the major virulence factors are expressed $\left(\mathrm{Bvg}^{+}\right.$phase), the flagellin gene is not and 
vice versa [13]. The bacterial flagellin gene has been used as a variable biomarker to study genetic variation amongst a number of bacterial pathogens [15], including Burkholderia cepacia [16] and Pseudomonas aeruginosa [17]. The study of variation within individual genes can provide an approach to analysing relationships between bacterial strains that is different from many molecular methods, which rely on whole genome analysis.

This study reports the development of a flagellin gene PCR-restriction fragment length polymorphism (RFLP) technique for $B$. bronchiseptica. This technique, coupled with nucleotide sequencing of B. bronchiseptica flagellin genes, was used to assess genetic variation and relationships between strains. The study of single gene variation was compared with whole genome variation as demonstrated by PFGE.

\section{Materials and methods}

\section{Bacterial strains}

The $B$. bronchiseptica isolates used in this study are listed in Table 1. All the isolates had been typed previously by PFGE after macro-restriction digestion with XbaI (Table 1) [10].

\section{PCR-RFLP analysis of B. bronchiseptica flagellin genes}

Oligonucleotide primers for amplification of $B$. bronchiseptica flagellin genes were designed from the published flaA coding sequence (1176 bp in length) for the strain GPISN [18]. A 1156-bp region of the flaA coding sequence (corresponding to a region of GPISN flaA from position 2 to 1157) was amplified by PCR with oligonucleotide primers BBFLAF: 5'TGGCTGCAGTCATCAATA-3' (N-terminal region, forward primer) and BBFLAR: 5'-ACGTTTTGCGGG ACTTGG-3' (C-terminal region, reverse primer), obtained from Sigma-Genosys (Cambridge). Strains were screened by first making a suspension of a few colonies in $20 \mu \mathrm{l}$ of sterile distilled water and boiling for $5 \mathrm{~min}$. This crude DNA $(1 \mu \mathrm{l})$ was used directly in $50-\mu 1$ volumes containing 2 units of Dynazyme (Flowgen Instruments, Sittingbourne, Kent), $200 \mathrm{nM}$ of each primer, $1 \times$ Dynazyme buffer and $100 \mu \mathrm{M}$ nucleotides (dATP, dCTP, dGTP, dTTP). Amplifications were performed in an OmniGene thermal cycler (Hybaid, Ashford, Middlesex) for 30 cycles consisting of $95^{\circ} \mathrm{C}$ $(1 \mathrm{~min}), 55^{\circ} \mathrm{C}(1 \mathrm{~min})$ and $72^{\circ} \mathrm{C}(2 \mathrm{~min})$. At the end of the amplification $10-\mu \mathrm{l}$ samples were subjected to electrophoresis on a standard agarose $1.0 \% \mathrm{w} / \mathrm{v}$ gel to confirm the presence of an amplified product. Samples of amplified product $(5 \mu \mathrm{l})$ were digested with the restriction endonucleases HaeIII, Msp I, Mbo I and $R s a \mathrm{I}$ with the conditions recommended by the supplier (Helena Biosciences, Sunderland, Tyne and Wear). These digests were then subjected to electrophoresis on MetaPhor agarose $1.5-2 \% \mathrm{w} / \mathrm{v}$ gels (Flowgen Instruments).

\section{Nucleotide sequencing and computer analyses}

Both strands of the PCR-amplified products of the flagellin gene were sequenced by the University of Liverpool DNA Sequencing Service with the primers BBFLAF and BBFLAR and other internal primers. Flagellin gene nucleotide sequences were aligned with each other or with other flagellin genes (retrieved from EMBL, GenBank or ftp://ftp.sanger.ac.uk/pub) by the programs GAP, PILEUP, FASTA and PRETTY from the GCG sequence analysis software package (Genetics Computer Group, University of Wisconsin, USA) [19].

\section{Nucleotide sequence accession numbers}

The nucleotide sequences obtained from the $\mathrm{flaA}$ genes of strains SB22, SB521 and SB283 have been assigned GenBank accession numbers AF232939-41.

\section{Results}

\section{PCR-RFLP of flagellin genes}

PCR amplicons of the flagellin genes were obtained from all the $B$. bronchiseptica isolates tested. Each of the amplicons was subjected to restriction digestion with four different endonucleases. Digestion with Hae III, Msp I and Rsa I each resulted in three different patterns, whereas only two patterns were obtained after digestion with MboI. The patterns obtained with an individual enzyme were designated $\mathrm{A}, \mathrm{B}$ or $\mathrm{C}$, depending on whether they were most common (A), second most common (B) or third most common (C). A gel showing examples of digests obtained with Hae III is shown in Fig. 1. Table 1 summarises the PCR-RFLP profiles of all the isolates, which fell into three groups. Each of these three groups could be distinguished by using Hae III, Msp I or Rsa I individually.

Of the 11 strains belonging to PFGE subtypes of strain A, five belonged to the most common flaA PCR-RFLP group (restriction pattern A with all four enzymes), with the other six belonging to the second most common PCR-RFLP group (restriction pattern B with all four enzymes). The distribution was less equal amongst the 10 strains belonging to PFGE subtypes of strain $\mathrm{B}$, eight of which belonged to the most common flaA PCR-RFLP group. Where PFGE subtypes were the same, PCR-RFLP analysis of the flagellin gene supported the relationship (two strains each of subtypes B3 and B7).

\section{Flagellin gene sequences}

Representative isolates from each of the three flaA PCR-RFLP groups (SB22, SB283 and SB521) were 
$M 12345678910 \quad M$

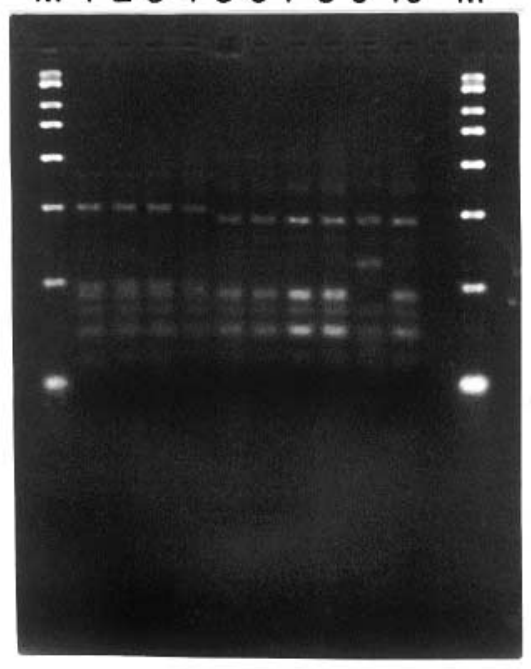

Fig. 1. Flagellin gene PCR products digested with Hae III. The gel shows a number of amplicons derived from different isolates of $B$. bronchiseptica, and subsequently digested with the endonuclease Hae III. Lane M, PCR size marker (fragment sizes 2000, 1500, 1000, 750, $500,300,150$ and $50 \mathrm{bp}$; R\&D Systems, Abingdon, Oxfordshire). Lanes 1-4, amplicons with PCR-RFLP pattern A; 5-8 and 10, amplicons with PCR-RFLP pattern $\mathrm{B} ; \mathbf{9}$, an amplicon with PCR-RFLP pattern C.

used to obtain flagellin gene sequences. These sequences were aligned with the previously published sequence from $B$. bronchiseptica strain GPISN [18] and the $B$. pertussis strain Tomaha I cryptic flagellin gene sequence (Fig. 2). The percentage identities for each pair of flagellin gene sequences were calculated over a region of the $\mathrm{flaA}$ gene corresponding to 1051 $\mathrm{bp}$ of the strain GPISN sequence (Table 2). Flagellin gene nucleotide sequences representing each of the three groups differed from each other by $11-13 \%$. By far the greatest variation was observed in the central region of the flaA gene (Fig. 2). The flaA sequence obtained from strain SB283 was $99.5 \%$ identical to the reported sequence for strain GPISN (100\% identical in predicted protein amino-acid sequence). Interestingly, both nucleotide and predicted amino acid sequence comparisons indicated that B. bronchiseptica SB283 and GPISN flagellin sequences are more closely related to the B. pertussis Tomaha I flagellin sequence than to the flagellin sequences of the other $B$. bronchiseptica isolates (Table 2).

\section{Discussion}

Isolates of B. bronchiseptica were separated into three groups by PCR-RFLP analysis of flagellin genes. Each of the groups gave different restriction digest patterns with at least three endonucleases, which means that the PCR-RFLP groups identified in this study can be distinguished by single enzyme digests with Hae III, $M s p$ I or RsaI. As the strains used in this study were resolved into 10 groups by macro-restriction analysis with $X b a$ I followed by PFGE, it is apparent that the

Table 1. Description of strains used in this study

\begin{tabular}{|c|c|c|c|c|c|c|}
\hline \multirow{2}{*}{$\begin{array}{l}\text { Isolate } \\
\text { no. }\end{array}$} & \multirow[b]{2}{*}{ Source } & \multirow{2}{*}{$\begin{array}{l}\text { PFGE } \\
\text { subtype* }^{*}\end{array}$} & \multicolumn{4}{|c|}{ Flagellin gene RFLP patterns } \\
\hline & & & Hae III & MboI & $M s p \mathrm{I}$ & $R s a \mathrm{I}$ \\
\hline SB22 & Cat & A1 & $\mathrm{B}$ & $\mathrm{B}$ & $\mathrm{B}$ & $\mathrm{B}$ \\
\hline SB55 & Cat & A6 & A & A & A & A \\
\hline SB48 & Cat & A10 & $\mathrm{A}$ & $\mathrm{A}$ & $\mathrm{A}$ & $\mathrm{A}$ \\
\hline SB80 & Cat & A11 & $\mathrm{B}$ & $\mathrm{B}$ & $\mathrm{B}$ & $\mathrm{B}$ \\
\hline SB101 & Cat & A12 & $\mathrm{B}$ & $\mathrm{B}$ & $\mathrm{B}$ & $\mathrm{B}$ \\
\hline SB110 & Cat & A13 & $\mathrm{B}$ & $\mathrm{B}$ & $\mathrm{B}$ & $\mathrm{B}$ \\
\hline SB116 & Cat & A19 & $\mathrm{B}$ & $\mathrm{B}$ & $\mathrm{B}$ & $\mathrm{B}$ \\
\hline SB200 & Cat & A22 & A & A & A & A \\
\hline SB193 & Cat & A24 & A & A & A & A \\
\hline SB197 & Cat & A26 & A & A & A & A \\
\hline SB236 & Cat & A 27 & B & B & B & B \\
\hline SB283 & Cat & B1 & A & A & A & A \\
\hline SB297 & Cat & B2 & A & A & A & A \\
\hline SB299 & Cat & B3 & A & A & A & A \\
\hline SB794 & Cat & B3 & A & A & A & A \\
\hline SB452 & Cat & B5 & $\mathrm{C}$ & A & $\mathrm{C}$ & $\mathrm{C}$ \\
\hline SB455 & Cat & B6 & B & B & B & B \\
\hline SB461 & Cat & B7 & A & A & A & A \\
\hline SB503 & Cat & B7 & A & A & A & A \\
\hline SB866 & Cat & B11 & A & A & A & A \\
\hline SB868 & Cat & B12 & A & A & A & A \\
\hline SB18 & Cat & $\mathrm{C} 1$ & B & B & B & B \\
\hline SB83 & Cat & E1 & B & B & B & B \\
\hline SB534 & Cat & E2 & B & B & B & B \\
\hline SB998 & Dog & H1 & A & A & A & A \\
\hline SB909 & Cat & I1 & A & A & A & A \\
\hline SB939 & Cat & $\mathrm{J} 1$ & A & A & A & A \\
\hline SB521 & Cat & $\mathrm{K} 1$ & $\mathrm{C}$ & A & $\mathrm{C}$ & $\mathrm{C}$ \\
\hline SB925 & Cat & L1 & A & A & A & A \\
\hline SB660 & Dog & N1 & A & A & A & A \\
\hline
\end{tabular}

${ }^{*}$ Determined in a previous study [10]. 


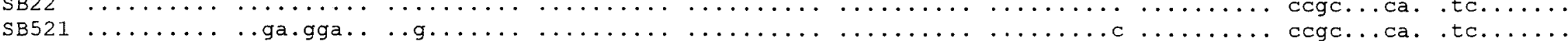

CON CCTGAAGTCC AACGCCACCG ACATGACCCT GTCGATCCAG GTCGGCGCCA AGGACAACGA AACGATCGAT ATCAAGATCG ATCGCAACTC GAACTGGAAC 451

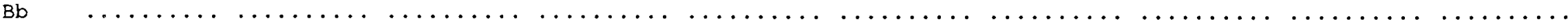

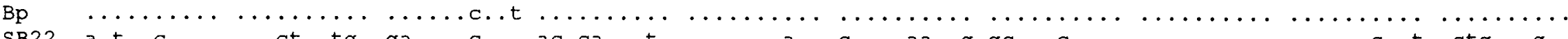

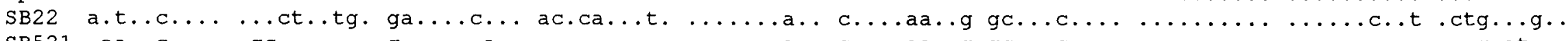

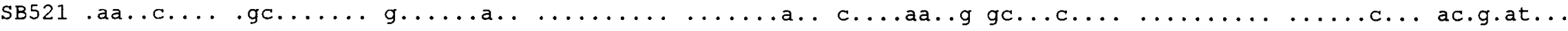

CON CTGTATGACG CCGTGGGCAC CGTCCCGGGC GGCACGGTCA ACGGCGAGGC TCGCACCGTC AACGCGCTGG GCTTTGACGT GCTGTCGGCC GTCACGACCA 551

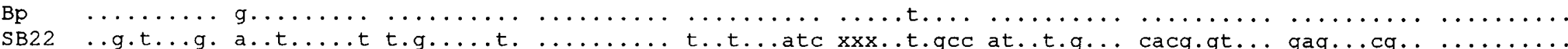

sB22 ..g.t..g. a.t....t t.g...t. .......t.t...atc xxx..t.gcc at..t.g...cacg.gt...gag...cg.........

SB521 .ggc..acgt g..ag..a.c t.g........t..c..ca...... xxx...aagc a.a......c.c.ac.a.. gt...aa.c a.......

CON CCATCGCTTC -GACACCGTG ACCTTCGACG CCGCCGTGGC GGCCGCCGAA CAGGCCGCTG GCGCCGCCGC AGGCGACGGC AGCGTCGTCT CGTACGGCGA 651

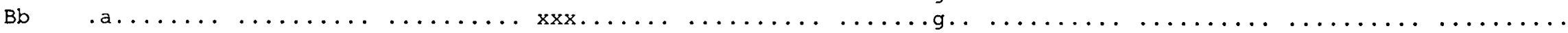

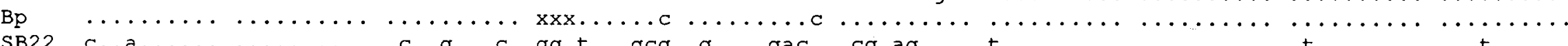

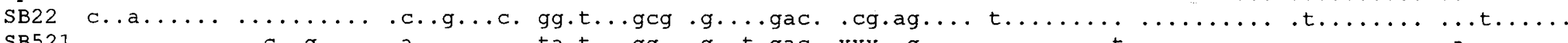

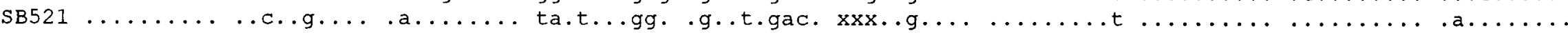

CON TGCCGCCAAC CCGCAATACG CGGTCGTGGT --CCGACAAT GCCGGCACGA TGACCTCCTA CGCCCTGACC TTCGACAAGG ACGGCAAGGC CGCCCTGGGC 751

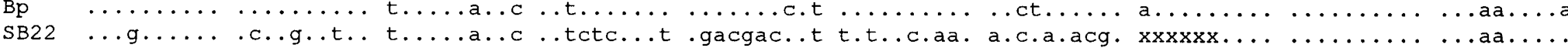
SB521 ..ag....c. .c..g.... ga......a ..c.cc..tt .gacgacg....t.ac.aat ...t.gacg. g..xxx.................

CON GACCAACTGG GTGCCGTCGC -TCGCAGGC- GC-GAAGCCG CCGTCGGTGC CAACGACGTC GCTGCCGGCG CCAACGTCAC CGTGTCCGGC GGCGCCGCCG

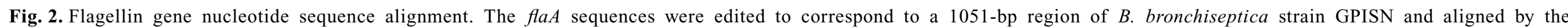

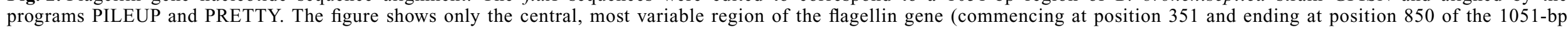

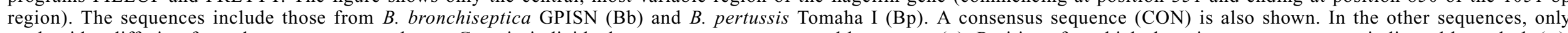

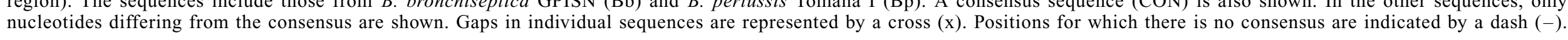


Table 2. Comparison of nucleotide sequences of flagellin genes

\begin{tabular}{lcccc}
\hline \multirow{2}{*}{$\begin{array}{l}\text { Flagellin sequence } \\
\text { of isolate no. }\end{array}$} & \multicolumn{4}{c}{ Percent identity* to flagellin sequence of strain } \\
\cline { 2 - 5 } & GPISN & SB22 & SB521 & SB283 \\
\hline SB22 & $87.9(86.5)$ & & & \\
SB521 & $87.2(83.9)$ & $88.7(86.4)$ & & \\
SB283 & $99.5(100)$ & $87.7(86.4)$ & $87.1(83.9)$ & \\
B. pertussis Tomaha I & $97.3(97.7)$ & $88.7(87.3)$ & $86.9(83.6)$ & $97.1(97.7)$ \\
\hline
\end{tabular}

*Levels of identity were determined over a 1051-bp region of $B$. bronchiseptica GPISN flaA sequence using the GAP program. Values in parentheses indicate comparisons between predicted amino-acid sequences for the equivalent region of $\mathrm{flaA}$.

flagellin gene PCR-RFLP method is not as discriminatory as PFGE. The isolates used in this study were chosen to include at least 10 representatives of each of the major PFGE types (A and B) identified in a previous study [10]. When the results from PFGE and flagellin gene PCR-RFLP were compared, there was no direct correlation between PFGE types and flaA PCRRFLP groups. Nucleotide sequence analysis confirmed the presence of three flagellin gene groups differing by $11-13 \%$ from each other.

Proteins present on the surface of bacterial cells, such as the flagellin protein, are known to be more variable than other bacterial proteins [20]. However, flagellin gene variation reflects the clonality of the organism under study. For example, in Burkholderia cepacia, flagellin genes are highly variable and can be readily separated into numerous PCR-RFLP groups [16, 21], whereas in Bur. pseudomallei very little flagellin gene variation is observed, especially amongst clinical isolates [22]. These observations correlate with the genetic variation observed in other approaches. In Bur. cepacia, genomic variation is so apparent that the species has been subdivided into genomovars [23]. In one study, the genetic relatedness of $B$. pertussis, $B$. bronchiseptica and $B$. parapertussis was examined by macro-restriction digestion of DNA with the rarely cutting endonucleases $X b a \mathrm{I}$ and resolution by PFGE. When the results of this work were compared with those of previous DNA-DNA hybridisation and multilocus enzyme electrophoresis studies, the authors concluded that these three species are so closely related that they should be considered members of the same species [9]. Indeed, flaA nucleotide sequence data suggest that the most common $B$. bronchiseptica flagellin gene group is more closely related to $B$. pertussis Tomaha I than to the other $B$. bronchiseptica flagellin gene groups. This has implications for the whole genome analysis following on from the completion of the genome sequences of $B$. pertussis Tomaha I and $B$. bronchiseptica RB50, the flaA sequence of which shares $99.9 \%$ identity with the flaA gene of GPISN (data not shown). Greater genetic variation may occur between strains currently designated as $B$. bronchiseptica than occurs between the strains of these two species chosen for whole genome sequencing. As has been observed in many other studies of flagellin genes, the variation is apparent mainly in the central region of the gene [15].

It is clear from this study that relationships between $B$. bronchiseptica isolates inferred by macro-restriction analysis do not agree with those calculated on the basis of sequence similarity in the flagellin genes. A recent study comparing the genomic organisation of 14 clinical isolates of $B$. pertussis revealed that whilst the genomic maps demonstrated general similarity in gene order, there were a number of examples of genomic re-arrangements in the form of large chromosomal inversions [24]. If such events were also found in B. bronchiseptica, then many of the current molecular methods for strain differentiation would discriminate between strains containing essentially the same genetic information. Whilst such genetic rearrangements would not invalidate the use of such techniques in epidemiological studies aimed at identifying identical strains, the possibility of such events suggests that relationships inferred from macro-restriction analysis by PFGE and other whole genome approaches should be treated with caution. The study of variations in other genetic loci in B. bronchiseptica will help to resolve the question of whether relationships inferred on the basis of flagellin gene sequences are more reliable than those calculated on the basis of restriction site variation detected by PFGE.

\section{References}

1. Goodnow RA. Biology of Bordetella bronchiseptica. Microbiol Rev 1980; 44: 722-738.

2. Woolfrey BF, Moody JA. Human infections associated with Bordetella bronchiseptica. Clin Microbiol Rev 1991; 4: 243255 .

3. Wright NG, Thompson H, Taylor D, Cornwell HJC. Bordetella bronchiseptica: a re-assessment of its role in canine respiratory disease. Vet Rec 1973; 93: 486-487.

4. Willoughby $\mathrm{K}$, Dawson $\mathrm{S}$, Jones RC et al. Isolation of $B$. bronchiseptica from kittens with pneumonia in a breeding cattery. Vet Rec 1991; 129: 407-408

5. Coutts AJ, Dawson S, Binns S, Hart CA, Gaskell CJ, Gaskell RM. Studies on natural transmission of Bordetella bronchiseptica in cats. Vet Microbiol 1996; 48: 19-27.

6. Jacobs AA, Chalmers WSK, Pasman J, van Vugt F, Cuenen LH. Feline bordetellosis: challenge and vaccine studies. Vet Rec 1993; 133: 260-263.

7. Keil DJ, Fenwick B. Evaluation of canine Bordetella bronchiseptica isolates using randomly amplified polymorphic 
DNA fingerprinting and ribotyping. Vet Microbiol 1999; 66: 41-51.

8. Register KB, Boisvert A, Ackermann MR. Use of ribotyping to distinguish Bordetella bronchiseptica isolates. Int $J$ Syst Bacteriol 1997; 47: 678-683.

9. Khattak MN, Matthews RC. Genetic relatedness of Bordetella species as determined by macrorestriction digests resolved by pulsed-field gel electrophoresis. Int J Syst Bacteriol 1993; 43: 659-664.

10. Binns SH, Speakman AJ, Dawson S, Bennett M, Gaskell RM, Hart CA. The use of pulsed-field gel electrophoresis to examine the epidemiology of Bordetella bronchiseptica isolated from cats and other species. Epidemiol Infect 1998; 120: 201-208.

11. Savelkoul PHM, de Kerf DPG, Willems RJ, Mooi FR, van der Zeijst BAM, Gaastra W. Characterization of the fim 2 and fim3 fimbrial subunit genes of Bordetella bronchiseptica: roles of Fim2 and Fim 3 fimbriae and flagella in adhesion. Infect Immun 1996; 64: 5098-5105.

12. Leigh AF, Coote JG, Parton R, Duggleby CJ. Chromosomal DNA from both flagellate and non-flagellate Bordetella species contains sequences homologous to the Salmonella H1 flagellin gene. FEMS Microbiol Lett 1993; 111: 225-231.

13. Akerley BJ, Monack DM, Falkow S, Miller JF. The $b v g A S$ locus negatively controls motility and synthesis of flagella in Bordetella bronchiseptica. J Bacteriol 1992; 174: 980-990.

14. Martinez de Tejada G, Miller JF, Cotter PA. Comparative analysis of the virulence control systems of Bordetella pertussis and Bordetella bronchiseptica. Mol Microbiol 1996; 22: $895-908$.

15. Winstanley C, Morgan JAW. The bacterial flagellin gene as a biomarker for detection, population genetics and epidemiological analysis. Microbiology 1997; 143: 3071-3084.
16. Hales BA, Morgan JAW, Hart CA, Winstanley C. Variation in flagellin genes and proteins of Burkholderia cepacia. $J$ Bacteriol 1998; 180: 1110-1118.

17. Winstanley C, Coulson MA, Wepner B, Morgan JAW, Hart CA. Flagellin gene and protein variation amongst clinical isolates of Pseudomonas aeruginosa. Microbiology 1996; 142: 2145-2151.

18. Akerley BJ, Miller JF. Flagellin gene transcription in Bordetella bronchiseptica is regulated by the BvgAS virulence control system. J Bacteriol 1993; 175: 3468-3479.

19. Pearson WR, Lipman DJ. Improved tools for biological sequence comparison. Proc Natl Acad Sci USA 1988; 85: 2444-2448.

20. Whittam TS. Genetic population structure and pathogenicity in enteric bacteria. In: Baumberg S, Young JPW, Wellington EMH, Saunders JR (eds) Population genetics of bacteria. 52nd Symposium of the Society for General Microbiology, University of Leicester, January 1995. Cambridge, Cambridge University Press. 1995: 217-245.

21. Winstanley C, Hales BA, Morgan JAW et al. Analysis of $\mathrm{fliC}$ variation among clinical isolates of Burkholderia cepacia. $J$ Med Microbiol 1999; 48: 657-662.

22. Winstanley C, Hales BA, Corkill JE, Gallagher MJ, Hart CA. Flagellin gene variation between clinical and environmental isolates of Burkholderia pseudomallei contrasts with the invariance among clinical isolates. J Med Microbiol 1998; 47: 689-694.

23. Vandamme P, Holmes B, Vancanneyt M et al. Occurrence of multiple genomovars of Burkholderia cepacia in cystic fibrosis patients and proposal of Burkholderia multivorans sp. nov. Int $J$ Syst Bacteriol 1997; 47: 1188-1200.

24. Stibitz S, Yang M-S. Genomic plasticity in natural populations of Bordetella pertussis. J Bacteriol 1999; 181: 5512-5515. 